Gabriela Marques-Schäfer*, Ebal Sant’Anna Bolacio Filho und Uwe Koreik

\title{
Aus- und Fortbildung von DaF-Lehrenden in Südamerika: Perspektiven und Herausforderungen
}

\author{
Einführung in den Themenschwerpunkt
}

https://doi.org/10.1515/infodaf-2020-0091

Im Zeitraum 2010-2015 war die Nachfrage für Deutsch als Fremdsprache in Südamerika groß. Gründe dafür waren vor allem die PASCH-Initiative und die Internationalisierungsbemühungen der Universitäten. Dazu war in den Ländern der Region festzustellen, dass die Nachfrage nach (weiteren) Fremdsprachen gestiegen war. Dies hing zweifelsohne mit dem in vielen Ländern zu beobachtenden wirtschaftlichen Wachstum zusammen (vgl. Auswärtiges Amt 2015). Zunehmend wurde Deutsch von Schülerinnen und Schülern sowie von Studentinnen und Studenten nach Englisch als zweite Fremdsprache gewählt. Insbesondere die Perspektive eines Studiums oder eines Forschungsaufenthaltes in Deutschland dürften hierfür verantwortlich sein. Ob dieser Trend weiterhin anhält, ist noch nicht klar, da sich die wirtschaftliche sowie die politische Lage in vielen südamerikanischen Ländern in den letzten zwei Jahren erheblich verändert hat. Laut dem vor kurzem erschienenen Bericht Deutsch als Fremdsprache weltweit. Datenerhebung 2020 ist seit dem letzten Bericht aus dem Jahr 2015 in Südamerika - zumindest in den zwei größten Ländern Argentinien und Brasilien - „ein leichter Rückgang zu beobachten; in den übrigen Staaten erhöhen sich die Zahlen moderat“ (Auswärtiges Amt 2020: 9).

Selbst wenn sich der leichte Rückgang in den beiden Ländern in den nächsten Jahren bestätigen sollte, lässt sich insgesamt in der Region aufgrund nicht genügender universitärer Lehrerausbildung für das Fach Deutsch als Fremdsprache ein Mangel an Lehrenden feststellen (vgl. Roche/Suñer 2017: 572). Bildungsund Förderinstitutionen haben in den letzten Jahren darauf reagiert, indem sie verstärkt in die Lehrerausbildung investieren und innovative Aus- und Fortbil-

*Kontaktpersonen: Gabriela Marques-Schäfer, E-Mail: gabrielamarques@yahoo.com Ebal Sant'Anna Bolacio Filho, E-Mail: ebolacio@gmail.com

Uwe Koreik, E-Mail: uwe.koreik@uni-bielefeld.de 
dungskonzepte und Internationalisierungsstrategien entwickeln, wie zum Beispiel in Brasilien (siehe dazu Mariano/Lorke in diesem Heft).

Vor diesem Hintergrund enstand die Idee, ein Themenheft zum Thema „Ausund Fortbildung von DaF-Lehrenden in Südamerika: Perspektiven und Herausforderungen" herauszugeben. Für das Themenheft waren Artikel erwünscht, die einen deutlichen Bezug zur Aus- und Fortbildung von DaF-Lehrkräften in den südamerikanischen Ländern haben und über reine Erfahrungsberichte hinausgehen. So freuten wir uns über viele Vorschläge, die von Kolleginnen und Kollegen aus Brasilien, Chile, Kolumbien und Paraguay eingereicht wurden.

Als wir die Idee für das Themenheft hatten, erwarteten wir Beiträge sowohl aus dem universitären Bereich als auch aus den zahlreichen Schulen der Region mit DaF-Unterricht, an denen auch viel über die Verbindung zwischen Theorie und Praxis nachgedacht wird. Unsere Erwartungen sollten sich erfüllen, so dass wir unter den insgesamt 15 eingereichten Vorschlägen leider eine schwierige Auswahl treffen mussten, um den Rahmen des Heftes nicht zu sprengen. Unseres Erachtens bieten die acht ausgewählten Beiträge dieses Themenheftes ein Panorama der Deutschlehrerausbildung in Südamerika, das aber natürlich keinen Anspruch auf Vollständigkeit hat.

Bei der Revision der Beiträge bestand unsere Aufgabe als Herausgeber hauptsächlich darin, die lateinamerikanische(n) Perspektive(n) nicht aus den Augen zu verlieren, um sie dem außenstehenden Leser verständlich zu vermitteln. Dies glauben wir dadurch erreicht zu haben, indem wir intensive fachliche Diskussionen mit den Autorinnen und Autoren führten - wofür wir uns an dieser Stelle bei den Kolleginnen und Kollegen ausdrücklich bedanken möchten.

Uns lag besonders am Herzen, vor allem die Perspektive aus der Fachdiskussion in den jeweiligen Ländern in den Vordergrund zu rücken. Es sollte nämlich nicht darum gehen, nur mit in deutschsprachigen Ländern verbreiteten Erkenntnissen zu arbeiten und aus dieser Perspektive heraus die lateinamerikanischen Kontexte zu beschreiben und zu analysieren, sondern einen Dialog zwischen den Erfahrungen und Erkenntnissen beider Kontinente zu etablieren.

Während wir dem vorliegenden Themenheft den letzten Schliff verpassen, tobt weltweit die Corona-Pandemie und bringt unweigerlich einschneidende Veränderungen in allen Lebensbereichen mit sich. Auch wenn das Ausmaß dieser Veränderungen momentan noch nicht vollständig abzusehen ist, steht eines jedoch bereits fest: Es werden Konsequenzen gezogen werden, auch wenn die Menschen nach überstandenen Schwierigkeiten gerne dazu tendieren, nach Möglichkeit so weiterzumachen wie zuvor.

Für das Lehren und Lernen (nicht nur) von Fremdsprachen werden aktuell unserer Kenntnis nach auch in Südamerika fast überall mehr oder weniger intensiv bereits verschiedene internetbasierte Werkzeuge sowie verschiedene Szena- 
rien ausprobiert. Wenn wir das Beispiel von Brasilien unter die Lupe nehmen, sehen wir, dass DaF-Lehrkräfte aufgrund der Pandemie in den Privatschulen, an denen Deutsch unterrichtet wird, sowie an Goethe-Instituten jetzt nur noch online unterrichten. Die meisten Lehrkräfte haben weder Erfahrung mit digitalen Medien noch mit internetbasierten fremdsprachlichen Fernkursen. Laut ihren Berichten arbeiten die Kolleginnen und Kollegen mit selbstgedrehten Video-Aufnahmen, PDFs, Power-Points und Materialien aus dem Internet.

In vielen Fällen geht es um eine praktische Übertragung des Präsenzunterrichts auf Online-Angebote, die über Apps und Plattformen wie Zoom®, GoogleMeet ${ }^{\circledR}$ und viele andere stattfinden. Das lässt sich kritisieren, aber ohne diese Zwangsdigitalisierung würden viele Privatschulen vor dem finanziellen Ruin stehen und viele DaF-Lehrkräfte würden zeitnah arbeitslos.

Auch wenn man schon seit Langem von „neuen Medien“ spricht, bekommt man dann den Eindruck, dass diese nicht wirklich in den Schulen und Universitäten - zumindest nicht in Brasilien - angekommen sind. Vielleicht erleben wir jetzt die Chance, digitale Medien tatsächlich verstärkt sinnvoll in den Unterricht $\mathrm{zu}$ integrieren, wobei die Betonung auf einer ausgeweiteten sinnvollen Integration liegen sollte und nicht auf dem schrittweise herbeigeführten Ersatz des Präsenzunterrichts und der Präsenzlehre, von der viele Bildungsplanerinnen und -planer weltweit zu träumen beginnen, weil sich damit unter anderem Kosten einsparen und Bildungsziele besser fokussieren und kontrollieren ließen.

Was diese gegenwärtig gemachten, großenteils erzwungenen Erfahrungen mit den digitalen Medien für die DaF-Lehrkräfteausbildung bedeuten, ist klar: Wir müssen uns mehr denn je um die Medienkompetenz unserer zukünftigen Kolleginnen und Kollegen bemühen. Denn die Pandemie zeigt uns unmissverständlich, dass es in Zukunft ohne einen sinnvollen Einsatz digitaler Medien und ohne die Fähigkeit, mit ihnen umzugehen, schwierig werden wird, als Lehrerin oder Lehrer sowohl in der Ausbildung als auch später auf dem Arbeitsmarkt zurechtzukommen bzw. sich zu behaupten.

Da dieses Themenheft den Ländern Südamerikas gewidmet ist, darf man ebenfalls die Tatsache nicht aus den Augen verlieren, dass in der Region immer noch eine riesige Kluft zwischen Arm und Reich besteht. Auch wenn die meisten Deutschlernerinnen und -lerner in Südamerika Privatschulen besuchen und im Prinzip keine finanziellen Schwierigkeiten mit den Kosten für Internetzugang und Beschaffung von Laptops, Handys sowie PCs haben dürften, gibt es auch öffentliche Institutionen wie Schulen und Universitäten, die Deutsch und andere Fremdsprachen anbieten, an denen sich Schülerinnen und Schüler bzw. Studentinnen und Studenten nicht leisten können, an digitalen Lernangeboten teilzunehmen. Und genau an solchen Situationen werden die Konsequenzen der 
Pandemie sichtbar: Die reichen Deutschlernenden können weiterhin Deutsch lernen, während die weniger Privilegierten das Nachsehen haben.

Es ist auch unklar, welche Konsequenzen die Corona-Pandemie auf Austauschprogramme, Lehrkräftefortbildungen und Fachtagungen haben wird. Wird auch hier mittelfristig mehr online durchgeführt werden? Erste weltweite Planungen für Deutschlehrkräftetagungen im Frühjahr 2021 deuten darauf hin: Deutsche Plenarrednerinnen und -redner werden ihre Beiträge live vom heimatlichen $\mathrm{Ar}$ beitszimmer aus liefern. Und das gibt dann auch noch das gute Gefühl, durch damit vermiedene Langstreckenflüge einen Beitrag zur Verhinderung der weiteren Klimaerwärmung geleistet zu haben. Ganz abgesehen davon, dass diese Variante für Veranstalterinnen und Veranstalter und die mit einbezogenen Mittlerorganisationen die kostengünstigere ist. Eine länger andauernde deutliche Reduktion von direkten Kontaktmöglichkeiten könnte spürbare Auswirkungen auf die zukünftige Rolle des Deutschen in Südamerika haben. In diesem Zusammenhang betonte Hellmann (2020), Direktor der DAAD-Außenstelle in Rio de Janeiro, vor Kurzem auf einer Online-Tagung die Wichtigkeit von Erfahrungen im Ausland - was zumindest hoffen lässt, dass der DAAD weiterhin in Austauschprogramme investieren wird.

Die in diesem Themenheft enthaltenen Beiträge behandeln verschiedene Aspekte der DaF-Lehrkräfteausbildung und ermöglichen dadurch einen Einblick in die sehr unterschiedlichen Kontexte innerhalb Südamerikas. Auffällig ist, dass nur die Beiträge aus Brasilien von Autorinnen und Autoren geschrieben wurden, deren Muttersprache nicht Deutsch ist. Es lässt sich daher fragen, warum dem so ist. Wird in den anderen südamerikanischen Ländern weniger auf Deutsch veröffentlicht? Wenn auf Deutsch veröffentlicht wird, wer schreibt - und kommt dabei aus welchem Fachbereich?

Diese und andere Fragen werden wir überhaupt nicht beantworten können, aber wir möchten an dieser Stelle dafür plädieren, dass insbesondere innerhalb des Fachs Deutsch als Fremdsprache in der Region mehr auf Deutsch veröffentlicht wird. Einerseits sind wir uns dessen bewusst, dass Portugiesisch und Spanisch als Amtssprachen selbstverständlich priorisiert werden, aber nur wenn häufiger auf Deutsch veröffentlicht wird, kann man den fachlichen Dialog mit den deutschsprachigen Ländern fördern und den Stellenwert der deutschen Sprache in Konkurrenz zum Englischen als Lingua franca unterstützen. Dabei sollte auch bedacht werden, dass die Reputation von Fremdsprachenlehrkräften immer auch von der sprachkompetenten zielsprachlichen fachlichen Auseinandersetzung in fachdidaktischen und fachwissenschaftlichen Fragestellungen abhängig ist, was im Übrigen oft auch erst den Zugang zur internationalen Zusammenarbeit ermöglicht.

Andererseits sind wir uns ebenfalls der Herausforderungen des wissenschaftlichen Schreibens in der Fremdsprache bewusst. Das ist gerade ein Grund mehr, 
um in die hiesige DaF-Lehrkräfteausbildung zu investieren, Sprachkurse auf fortgeschrittenen Niveaus häufiger anzubieten - sowohl an den Hochschulen als auch an (Sprach-)Schulen. Dadurch könnte man die Präsenz der deutschen Sprache in der Region verstärken.

Damit möchten wir keinesfalls die Mehrsprachigkeit in Frage stellen. In einigen Fachzeitschriften, wie zum Beispiel Projekt des brasilianischen Deutschlehrerverbandes und Pandaemonium Germanicum der Universidade de Sao Paulo (Texte auf Spanisch und Englisch werden ebenfalls veröffentlicht), darf man zum Beispiel sowohl auf Deutsch als auch auf Portugiesisch veröffentlichen - und es soll so bleiben. Andere Zeitschriften wiederum veröffentlichen in speziellen (Themen-)Heften auch Texte auf Deutsch, wie zum Beispiel die zwei Zeitschriften Linguagem e Ensino und Caderno de Letras der südbrasilianischen Universidade Federal de Pelotas.

Wir sind der Meinung, dass eine sehr enge Zusammenarbeit zwischen südamerikanischen und deutschsprachigen Hochschulen - aber auch Schulen - vonnöten ist, um den Wissensaustausch im Germanistik-/DaF-Bereich noch weiter voranzutreiben. In diesem Zusammenhang spielen Förderagenturen eine sehr wichtige Rolle, um Programme - wie zum Beispiel die vom DAAD im universitären Bereich finanzierten bzw. kofinanzierten „Germanistischen Institutspartnerschaften“ (GIP) und „German Teachers Assistants“ (GTAs in Brasilien, zusammen mit der brasilianischen Förderagentur CAPES) - zu etablieren bzw. auszuweiten, damit Deutschlernende einen direkten Kontakt zu einer Fachperson aus dem deutschsprachigen Raum haben, die das Lehren und Lernen der deutschen Sprache in verschiedenen Kontexten unterstützen kann.

\section{Zu den Beiträgen}

In diesem Heft finden die Leserinnen und Leser acht Beiträge aus drei verschiedenen Ländern: Chile, Kolumbien und Brasilien. Mit dem einzigen Beitrag aus Chile beschreibt Sara Moldenhauer, die seit 2017 als DAAD-Lektorin an der Pontificia Universidad Católica de Valparaíso in Chile tätig ist, die DaF-Landschaft in der Region Valparaíso und stellt fest, dass dort in den letzten Jahren ein massiver Rückgang der Nachfrage hinsichtlich Deutsch als Fremdsprache zu beobachten ist. Zusätzlich zu einem detaillierten Überblick über die aktuelle Situation in der Region um Valparaíso versucht sie rückblickend darzustellen, wie die Entwicklung von einer der früher aktivsten Regionen in der Aus- und Fortbildung von DaF-Lehrkräften in Lateinamerika hin zu der aktuellen prekären Lage vonstatten ging. Abschließend werden Versuche vorgestellt, die momentan unternommen werden, um dieser traurigen Situation entgegenzuwirken. 
Die Autorinnen Zuzana Münch-Manková (Universität Göttingen) und Juliane Müller de Acevedo (Goethe-Institut Saõ Paulo) befassen sich mit der kritischen Darstellung der Fortbildung „Sprachbildung und -förderung / Deutsch als Zweitund Fremdsprache“, im Rahmen derer kolumbianische und deutsche Lehrkräfte gemeinsam weitergebildet worden sind. Neben den sprachpolitischen Grundlagen des DaZ-Unterrichts sowie der Förderung bildungssprachlicher und interkultureller Kompetenzen setzten sich die Teilnehmerinnen und Teilnehmer mit dem Konzept des Sprachsensiblen Fachunterrichts sowie der Sprachdiagnostik auseinander. Dabei ist als besonders wichtig hervorzuheben, dass diese Zusatzqualifikation von der Universidad Nacional de Colombia (UNAL) und den Unterrichtsbegleiterinnen und -begleitern der deutschen Schule Colegio Andino analysiert und diskutiert wurden, um die Inhalte und Methoden, die ursprünglich in Deutschland entwickelt worden waren, den kolumbianischen Bedürfnissen anzupassen. Ohne Zweifel zeigt die im Beitrag dargestellte Erfahrung ein gutes Beispiel von gelungener Synergie zwischen theoretischen Inhalten und Unterrichtspraxis.

Anelise F. P. Gondar und Mergenfel Vaz Ferreira arbeiten im brasilianischen Bundesstaat Rio de Janeiro und befassen sich mit dem Thema der Professionsbildung in der DaF-Lehrkräfteausbildung. Nach einem exploratorischen Exkurs in die Fachliteratur zum Thema Professionalisierung von Fremdsprachenlehrerinnen und -lehrern im brasilianischen Kontext präsentieren und diskutieren die Autorinnen die subjektiven Theorien im Rahmen der Berufsidentitätsforschung. Anhand einer Befragung unter den Studentinnen und Studenten des Doppelstudiengangs Deutsch-Portugiesisch an beiden Universitäten werden die Meinungen der Absolventinnen und Absolventen erfasst und analysiert, um einen besseren Einblick in den Stand der subjektiven Professionsbildung brasilianischer Studentinnen und Studenten zu bekommen.

Kristina Müller, Luisa Friederici und Sophie von Werder sind in Kolumbien an verschiedenen Institutionen im DaF-Bereich tätig. Die Autorinnen arbeiteten gemeinsam im Rahmen des deutsch-kolumbianischen Doppelmasterprogramms „Maestría en Lingüística - Linea Alemán como Lengua Extranjera/MA. Deutsch als Zweit- und Fremdsprache“ an der Universidad de Antioquia in Medellín, Kolumbien. In ihrem Beitrag präsentieren sie eine Bilanz des erwähnten Masterstudiengangs, die auf der Analyse der Daten einer Befragung unter Absolventinnen und Absolventen fußt. Erfolge und Herausforderungen des Programms werden diskutiert. Die Ergebnisse dieser Diskussion sollen als Grundlage für die Verbesserung dieses wichtigen Fortbildungsangebotes in Lateinamerika dienen.

Manuela Vogelgesang aus Kolumbien beschreibt das Konzept und die Entstehung des Unterrichtstrainer-Konzepts der Deutschen Schule Medellín (DSM). Es werden wichtige Erfolge eines schulinternen Lehrkräftefortbildungskonzepts dar- 
gestellt und diskutiert. Darüber hinaus präsentiert die Autorin aktuelle Entwicklungslinien und Herausforderungen, die zu mehr Unterrichtsqualität und Nachhaltigkeit in der schulinternen (DaF-/DFU-) Lehrkräftefortbildung führen.

Tito Lívio Cruz Romão, Rogéria C. Pereira und Francisco Gleiberson dos Santos Nogueira aus dem nordöstlichen brasilianischen Bundesstaat Ceará sind an der Universidade Federal do Ceará (UFC) tätig und beschäftigen sich mit der Frage der Stellung der Phonetik in der DaF-Lehrkräfteausbildung. Nach einer Revision der wissenschaftlichen Werke, die Lehrenden zum Thema kontrastive Phonetik (Deutsch/brasilianisches Portugiesisch) von Nutzen sein könnten, befassen sich die Autoren und die Autorin mit grundlegenden Schwerpunkten des Seminars „Phonetik und Phonologie des Deutschen“ an der UFC. Zum Schluss wird die Problematik der Phonetik-Darstellung in DaF-Lehrwerken im Laufe der letzten 25 Jahre zusammengefasst.

Franziska Lorke und Thiago Viti Mariano sind an der Universidade Federal Tecnológica do Paraná (UFTPR) bzw. an der Universidade Federal do Paraná (UFPR) im südbrasilianischen Bundesstaat Paraná tätig. Ihr Artikel behandelt die Maßnahmen zur DaF-Lehrkräfteausbildung, die in den letzten drei Jahren im Rahmen von einem Internationalisierungsprogramm der brasilianischen Regierung (Idiomas sem Fronteiras, kurz IsF) an beiden Universitäten konzipiert und durchgeführt wurden. Beschrieben und diskutiert werden die im Rahmen des IsF-Programms entwickelten Fortbildungsmaßnahmen, die das Ziel haben, Lehramtsstudentinnen und -studenten nicht nur Erfahrungen mit Blended-Learning-Szenarien $\mathrm{zu}$ ermöglichen, sondern auch die Verbindung zwischen theoretischen Reflexionen und praktischen Anwendungen zu fördern.

Der letzte Beitrag dieses Hefts wurde von Gabriela Marques-Schäfer und Ebal Sant'Anna Bolacio Filho verfasst, um eine vom DAAD finanzierte Studienreise für brasilianische DaF-Studierende zu evaluieren. Anhand einer Analyse von Daten, die mit Hilfe eines Fragebogens erhoben wurden, werden Aspekte der Planung und Organisation sowie des inhaltlichen Programms und der individuellen sprachlichen und interkulturellen - Erfahrungen der teilnehmenden Studierenden der Reise diskutiert. Dabei ging es der Forscherin und dem Forscher vor allem um die Frage, welche Rolle die Reise für den beruflichen, akademischen und persönlichen Wedergang der Teilnehmerinnen und Teilnehmer gespielt hat. Sie unterstreichen die Wichtigkeit einer solchen Initiative, um die Motivation angehender DaF-Lehrerinnen und -Lehrer für das Fach und für den Beruf des Lehrers bzw. der Lehrerin zu fördern. 


\section{Literatur}

Auswärtiges Amt (Hrsg.) (2015): Deutsch als Fremdsprache weltweit. Datenerhebung 2015.

Online: https://www.dw.com/downloads/29827615/statistik-2015-deutschlerner-weltweit. pdf (01.07.2020).

Auswärtiges Amt (Hrsg.) (2020): Deutsch als Fremdsprache weltweit. Datenerhebung 2020. Online: https://www.auswaertiges-amt.de/blob/2344738/b2a4e47fdb9e8e2739bab2565f8fe7c2/ deutsch-als-fremdsprache-data.pdf (01.07.2020).

Hellmann, Jochen (2020): „Is international experience really only a click away?“. Vortrag auf der Online-Tagung Academic Mobility and Cooperation in the Digital Age: Manifold Potentials and Challenges am 29.04.2020. Online: https://www.daad.org.br/files/2020/04/Jochen Hellmann_DAAD.pdf (12.06.2020).

Mariano, Thiago; Lorke, Franziska (2020): „Das Programm Deutsch ohne Grenzen in Brasilien: Chancen und Herausforderungen für die Ausbildung von Deutschlehrerinnen und Deutschlehrern“. (In diesem Heft).

Roche, Jörg; Suñer, Ferran (2017): „DaF-Kompetenzzentren: Chancen und Perspektiven der Deutschlehrerausbildung in Südamerika. Ein Bestands- und Entwicklungsbericht“. In: InfoDaF 41 (5), 571-580. 